\title{
ON THE FORMATION OF LINE PROFILES OF SOLAR P MODES
}

\author{
I.W. ROXBURGH ${ }^{1}$ AND S.V. VORONTSOV ${ }^{1,2}$ \\ ${ }^{1}$ Astronomy Unit, Queen Mary and Westfield College \\ Mile End Road, London E1 4NS, UK; \\ ${ }^{2}$ Institute of Physics of the Earth \\ B.Gruzinskaya 10, Moscow 123810, Russia
}

\begin{abstract}
We address the problem of the opposite asymmetry of low-frequency p-mode line profiles observed in intensity and velocity measurements (Duvall et al. 1993). We use a simple model to illustrate that this feature can be explained by including a contribution from the stochastic excitation velocity field to the non-resonant background in the doppler measurements.
\end{abstract}

Our analysis is based on the simple model of acoustic power spectra described in Roxburgh and Vorontsov (1995). We extend this theoretical description slightly, allowing for an instantaneous point-like source - an excitation which is described by $\delta$-function in both space and time. We make a simple order of magnitude esimate of the source function for monopole and dipole excitations, and also estimate the direct contribution to the doppler velocity signal from the kinetic velocity of the convective eddy (Roxburgh and Vorontsov 1997). We define the total complex amplitude $A(\omega)$ of the doppler velocity measurements as

$$
A(\omega)=k_{m o n} A_{m o n}(\omega)+k_{d i p} A_{d i p}(\omega)+k_{k i n} A_{k i n}(\omega),
$$

with three weighting coefficients $k_{m o n}, k_{d i p}$ and $k_{k i n}$. The first two coefficients are the efficiency factors of monopole and dipole excitation; $k_{k i n}$ is a factor of "visibility" of the kinematic velocity field. When the kinematic effect is neglected, the line profiles seen in intensity and in doppler measurements are similar. We expect the intensity data to be less sensitive to the kinematic velocity field, and simulate the results of the intensity measurements simply by using the Eq.(1) with $k_{k i n}$ set to zero. We also expect the efficiency factors to have nearly the same order of magnitude, if our hypothesis is correct.

Fig.1 shows the power spectrum produced by a composite excitation source. Line profiles depend on the relative magnitude and phase of the even and odd components of the excitation; the observational asymmetry of intensity measurements (smaller amplitude at the lower-frequency wing of the line) can be easily reproduced. The results obtained with the same composite excitation source but with the kinematic effect taken into account are shown in Fig.2. The line asymmetry is changed by the coherent contribution to the acoustic background of the kinematic-velocity signal. 


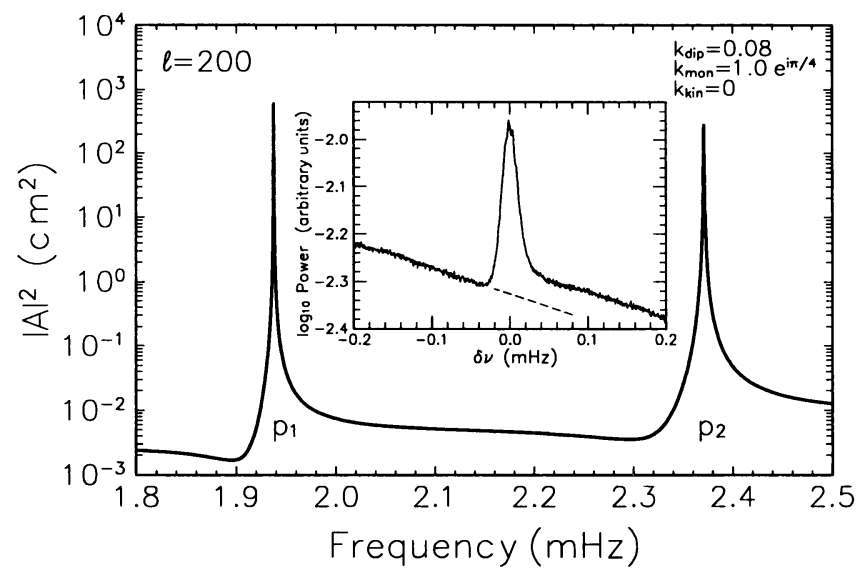

Figure 1. Artificial power specrum for a composite excitation source. Line profile observed in the intensity measurements (Duvall et al. 1993) are shown in the insert (courtesy S.M. Jefferies)

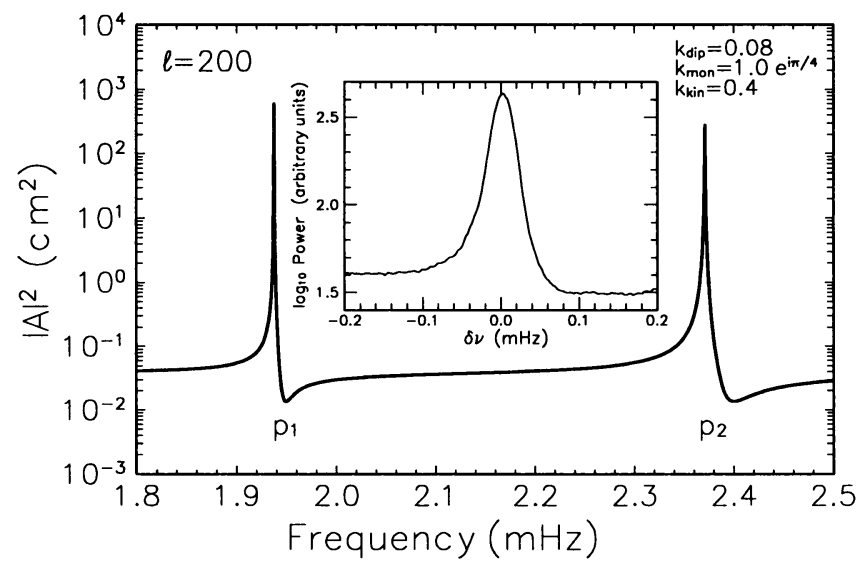

Figure 2. Same as Fig.1, but with additional contribution of the kinematic effect. The insert shows the line profile observed in velocity measurements (Duvall et al. 1993, courtesy S.M. Jefferies)

\section{References}

Duvall, T. L., Jr., Jefferies, S. M., Harvey, J. W., Osaki, Y. and Pomerantz, M. A. (1993), ApJ 410, p.829

Roxburgh, I. W. and Vorontsov, S. V. (1995), Mon. Not. R. astr. Soc. 272, p.850

Roxburgh, I. W. and Vorontsov, S. V. (1997), Mon. Not. R. astr. Soc., in press 